\title{
ANÁLISIS DE FITOESTEROLES EN LA SEMILLA DE Persea americana Miller (Var. Lorena) POR CROMATOGRAFÍA DE GASES Y CROMATOGRAFÍA LÍQUIDA DE ALTA EFICIENCIA
}

\section{ANALYSIS OF PHYTOSTEROLS IN SEEDS OF Persea americana Miller (Var. Lorena) BY GAS CHROMATOGRAPHY AND HIGH PERFORMANCE LIQUID CHROMATOGRAPHY}

\author{
Rosa Stefania BARRERA LÓPEZ1, Juan Pablo ARRUBLA VÉLEZ* \\ Grupo de investigación de Oleoquímica, Escuela de Tecnología Química, Facultad de \\ Tecnología, Universidad Tecnológica de Pereira, Pereira, Colombia. \\ *Autor Corresponsal. E-mail: juanpablo77@utp.edu.co
}

Historia del Artículo

\section{Resumen |}

\begin{abstract}
La presente investigación permitió determinar el contenido y composición de fitoesteroles en el aceite de la semilla de Persea Americana Miller variedad Lorena. La extracción soxhlet (SLE) tuvo un rendimiento de $8.47 \pm 0.084 \%$, la saponificación asistida por microondas y limpieza por extracción en fase sólida (MW-SPE) tuvo porcentajes de recuperación de $86.22 \pm 0.020 \%$. Se logró cuantificar el estigmasterol con $19.17 \pm 0.020 \mathrm{ppm}$ y el $5 \alpha$-colestano con una concentración de $49.77 \pm 0.020$ ppm. La confirmación para compuestos sin estándar se llevó a cabo utilizando detector de masas (MSD) encontrando derivados de 5-a-colestano. Para el análisis de ergosterol se utilizó el método de Cromatografía Líquida de Alta Eficiencia (CLAE). El aceite obtenido a partir de las semillas de Persea americana Miller variedad Lorena demostró la presencia de contenidos apreciables de fitoesteroles, indicando su uso potencial como insumo de formulaciones farmacéuticas así como en la fabricación de productos nutracéuticos y cosmecéuticos.
\end{abstract}

Palabras Clave: Aceite, Aguacate, Fitoesteroles, Saponificación, Soxhlet.

\section{Abstract}

The present investigation enabled to determine the phytosterols content and their composition in the oil of the seed of Persea americana Miller Lorena variety. The soxhlet extraction (SLE) had a yield of $8.47 \pm 0.084 \%$, microwave assisted saponification and solid phase extraction cleaning (MW-SPE) had percentages recovery of $86.22 \pm 0.020 \%$. Stigmasterol was quantified with $19.17 \pm$ $0.020 \mathrm{ppm}$ and 5 - $\alpha$-cholestane at a concentration of $49.77 \pm 0.020 \mathrm{ppm}$. Confirmation for without standard compounds it has been made using mass detector (MSD) it was found 5 - $a$-cholestane derivatives. High-Performance Liquid Chromatography (HPLC) method was used for the ergosterol analysis. The oil obtained from the seeds of Persea americana Miller Lorena variety showed the presence of appreciable phytosterols contents, indicating their potential use the input of pharmaceutical formulations as well as in the manufacturing of nutraceutical and cosmeceutical products.

Keywords: avocado, Oil, phytosterols, saponification, soxhlet.

\section{INTRODUCCIÓN |}

El aguacate pertenece a una de las familias más primitivas de las dicotiledóneas las Lauráceas, está compuesta por 52 géneros y cerca de 3.500 especies. El género Persea está formado por 150 especies distribuidas en las regiones tropicales y subtropicales, especialmente en Asia, Islas Canarias y América, donde existen 80 especies (Bernal y Díaz, 2008). El aguacate es una baya con mesocarpio y endocarpio carnoso que contiene una sola semilla ( 15 al $16 \%$ del peso del fruto), se caracteriza por el contenido de ácidos grasos insaturados principalmente monoinsaturados; es rico en vitamina $A, B$ y $E$, ácido ascórbico, $\beta$-caroteno, potasio y magnesio (Ozdemir y Topuz, 2004), sin embargo, la composición, la síntesis de metabolitos secundarios y las propiedades nutricionales de los frutos son muy variables y se ven influenciadas por factores como el clima, el suelo, la temperatura, la humedad, la cantidad de lluvia durante el desarrollo del fruto $y$ 
diferencias genotípicas entre variedades (Howard et al., 2003; Thomas et al., 2005).

El fruto Persea americana Miller variedad Lorena fue originado en la finca Lorena, en Palmira, Valle del Cauca, Colombia, en 1957. La época de cosecha en Colombia es de mediados de noviembre a febrero y de abril a julio. La relación cáscara: semilla: pulpa es 3:10:87\%, respectivamente (Bernal y Díaz, 2008). Este fruto se cultiva muy bien a bajas altitudes, aunque ha tenido buen comportamiento en las zonas cafeteras de Colombia (1.500 m.s.n.m). En el país se conoce una selección de Lorena denominada como aguacate Papelillo, que está bastante difundido en zonas medias y cálidas, con muy buen mercado por su calidad interna tanto para consumo en fresco como para su transformación (Bernal y Díaz, 2008). Presenta frutos de forma alargada, ligeramente oblicuos; corteza lisa, lustrosa, abundante punteado o número de nucelas; frutos de tamaño grande, peso aproximado de $400 \mathrm{~g}$, contenido de grasa del $9 \%$, color verde y pedúnculo largo. La semilla es de tamaño mediano, ovoide y simétrico, con mediana adherencia a la pulpa (Bernal y Díaz, 2008), sin embargo, después de la transformación industrial, aprovechamiento o consumo del fruto, se tienen materiales residuales principalmente semilla y epicarpio que podrían ser fuente potencial para complementos alimenticios por el contenido de metabolitos con diferentes actividades biológicas (Polania, 2014)

Actualmente, la semilla representa un recurso infrautilizado y es tema de preocupación en cuanto a su disposición final por la alta generación en las industrias procesadoras de aguacate. Estudios fitoquimicos previos realizados a la semilla del aguacate han permitido identificar diferentes clases de metabolitos entre los que se encuentran glucósidos del ácido abscísico, fitoesteroles, triterpenos, ácidos grasos, ácidos furanoicos, dímeros de flavanoles, proantocianidinas oligoméricas, polifenoles y antocianinas (Polania, 2014). Existe información de etnofarmacología, especialmente en los países de América del Sur sobre el uso de estas semillas para el tratamiento de ciertas enfermedades, donde en la actualidad se cultiva en gran escala. Recientemente se ha encontrado que las semillas de aguacate pueden mejorar la hipercolesterolemia, y ser útiles en el tratamiento de la hipertensión, afecciones inflamatorias, diabetes y osteoartítaricas. También se han encontrado que poseen actividad insecticida, fungicida y antimicrobiana. Históricamente se utilizaron extractos de semillas de aguacate como tinta para la escritura, junto al tinte producido cuando se usa una polifenol oxidasa sobre los mismos extractos (Polania, 2014).

Los fitoesteroles son esteroles de origen vegetal con estructura química similar y funciones análogas al colesterol, que difieren únicamente en la presencia de sustituyentes (Piironen et al., 2000; Moreau et al., 2002; Valenzuela y Ronco, 2004; Moreau y Hicks, 2005) y se pueden encontrar en forma libre o conjugados (Moreau et al., 2002; Nystrom et al., 2005). En los últimos años, los fitoesteroles han sido objeto de múltiples investigaciones debido a sus propiedades biológicas, destacándose: propiedades antiinflamatorias (Michelle y Manohar, 2009), antitumorales, antiaterosclerótica (Moghadasian y Frohlich, 1999), anticancerígenas (Awad y Fink, 2000), antioxidante (Wang et al., 2002) y antifúngicas (Smania et al., 2003), sin embargo, el efecto mejor caracterizado es el hipocolesterolémico (Moreau et al., 2002; Brufau et al., 2008).

Se han descrito más de 200 tipos de esteroles vegetales en diferentes especies de plantas, siendo los más abundantes 6 -sitosterol, campesterol y stigmasterol (Fig. 1), constituyendo el $95-98 \%$ de los fitoesteroles identificados. Se encuentran distribuidos en tallos, frutos y hojas vegetales. Las principales fuentes son los aceites vegetales, semillas oleaginosas, cereales, legumbres y frutos secos (Valenzuela y Ronco, 2004).

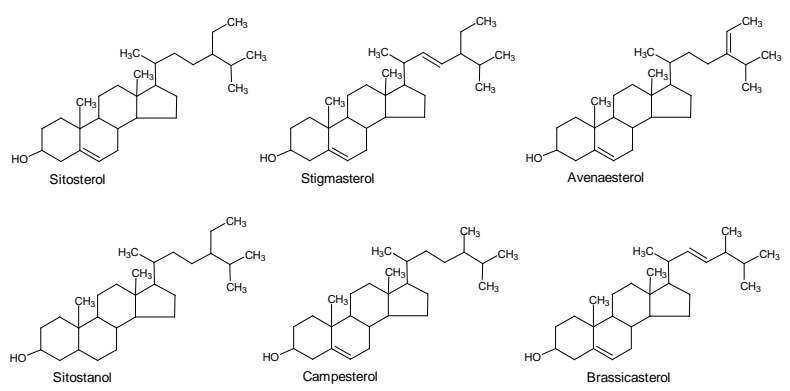

Figura 1. Estructura química de fitoesteroles y fitoestanoles.

Debido a que los fitoesteroles tienen una complejidad estructural y semejanza, las técnicas analíticas para el aislamiento de esteroles, separación y cuantificación deben ser precisas y exactas (Verleyen et al., 2002; Dutta et al., 2005; Harrabi et al., 2008). Algunas de las técnicas utilizadas para la identificación y cuantificación de estos compuestos en diferentes matrices (Abidi, 2001; Lagarda et al., 2006) son Cromatografía de Gases (CG) con detección de ionización de llama (FID) y/o detección de espectrometría de masas (MS) (Toivo et al., 2001; Santos et al., 2007; Clement et al., 2010) y Cromatografía Líquida de Alta Eficiencia (CLAE) (Abidi, 2001). Además se ha utilizado en combinación estas dos técnicas (CG y CLAE) con sistemas diferentes de detección para el análisis de fitoesteroles en los alimentos de alto rendimiento (Lagarda et al., 2006; Slavin y Yu, 2012).

Poco se ha investigado sobre la distribución del contenido de fitoesteroles en la semilla de Persea americana Miller variedad Lorena; por tanto, el objetivo de este trabajo fue estandarizar un método y cuantificar los principales fitoesteroles en la semilla, para ello, se desarrolló un método de extracción de aceite, separación de la fracción de fitoesteroles en la materia insaponificable del aceite y posterior identificación y cuantificación de fitoesteroles libres y conjugados, con el fin de mejorar el área de conocimiento del fruto, su aprovechamiento y propiciar apoyo para nuevas investigaciones de aplicación. 


\section{MATERIALES Y MÉTODOS}

\section{Estándares}

Se utilizaron cuatro estándares de referencia de fitoesteroles marca SUPELCO® (Tabla 1).

\begin{tabular}{ccc} 
& \multicolumn{3}{c}{ Tabla 1. Estándares de fitoesteroles. } \\
\hline Referencia & Fitoesteroles & Concentración \\
\hline $481-21-0$ & 5 a-Colestano & $10 \mathrm{mg} / \mathrm{mL}$ \\
$57-87-4$ & Ergosterol & $10 \mathrm{mg} / \mathrm{mL}$ \\
$83-48-7$ & Estigmasterol & $10 \mathrm{mg} / \mathrm{mL}$ \\
$80-97-7$ & Colestanol & $10 \mathrm{mg} / \mathrm{mL}$ \\
\hline
\end{tabular}

Tratamiento de las semillas previo a la extracción

Se utilizaron frutos de Persea americana Miller variedad Lorena (Fig. 2), obtenidos del municipio de Marsella en el departamento de Risaralda, Colombia. Los frutos fueron transportados en bolsas plásticas al laboratorio de Oleoquímica de la Universidad Tecnológica de Pereira, donde se dejaron madurar por un tiempo para posteriormente realizar la extracción de las semillas.

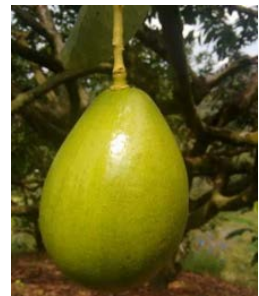

Figura 2. Fruto de Persea americana Miller variedad Lorena del Municipio de Marsella.

Las semillas fueron sometidas a un proceso de limpieza y lavado con detergente TEGO 51, luego se trituraron y se secaron en una estufa durante 48 horas a $45^{\circ} \mathrm{C}$, terminado este proceso se realizó la molienda hasta obtener un polvo fino (Fig. 3) y por último se almacenaron en bolsas plásticas herméticas, a una temperatura de $4^{\circ} \mathrm{C}$ para posterior extracción del aceite, de acuerdo con estudios anteriores (Arias y Gil, 2012).

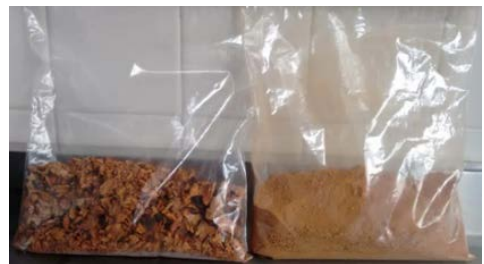

Figura 3. Semillas trituradas y molidas de Persea americana Miller variedad Lorena

\section{Extracción de aceite}

La extracción del aceite de semillas de Persea americana Miller, se realizó por el método soxhlet, utilizando como solvente $n$-hexano en una relación muestra: solvente (1:5), por un periodo de 24 horas, concentrando el extracto por rotaevaporación, según estudios previos (Gaona, 2011). El aceite obtenido fue almacenado en un recipiente ámbar a temperatura ambiente.

\section{Saponificación de la muestra}

La muestra de aceite (aproximadamente 10-100 mg), se colocó en un frasco tapa rosca, se agregó $10 \mathrm{~mL}$ de una solución metanolica de hidróxido de potasio $2 \mathrm{M}$. El frasco cerrado herméticamente se colocó en el centro de un horno microondas doméstico y se calentó a una potencia de 50\% durante $35 \mathrm{~s}$, después de $15 \mathrm{~min}$ se sometió al mismo calentamiento para terminar la saponificación del aceite y pasados 10 min se neutralizó con una solución acuosa de ácido clorhídrico 1 M (Young, 1995).

\section{Extracción de materia insaponificable}

Se realizó una extracción líquido-líquido inicialmente con $10 \mathrm{~mL}$ de $\mathrm{n}$-hexano, seguida de dos extracciones más con 5 $\mathrm{mL}$ de $n$-hexano (Young, 1995), se recogió la fase orgánica de cada extracción y se concentró la materia insaponificable.

\section{Extracción de fracción de fitoesteroles}

Se realizó una extracción en fase sólida para la obtención de la fracción de fitoesteroles a partir de la materia insaponificable, donde se utilizaron Sep-Pak de $\mathrm{Si}-\mathrm{OH}$ (fase normal) de $200 \mathrm{mg} / 3 \mathrm{~mL}$, como eluyentes $10 \mathrm{~mL}$ de una mezcla hexano:acetato de etilo (90:10) para la fracción no polar (fitoesteroles esterificados) y $10 \mathrm{~mL}$ de una mezcla etanol:éter etílico:hexano (50:25:25) para la fracción polar (fitoesteroles libres) (Rodríguez et al., 2015), las fracciones se mezclaron y se concentraron en un rotaevaporador.

\section{Derivatización de la fracción de fitoesteroles}

La muestra se derivatizó con $150 \mu \mathrm{L}$ de SILYL-991 (BSTFA-TMCS, 99:1), se agitó por 3 min en un vortex y se colocó en un baño de agua a $70^{\circ} \mathrm{C}$ por 30 minutos (Arias y Gil, 2012).

\section{Porcentaje de recuperación}

El porcentaje de recuperación de la técnica de extracción asistida por microondas, se determinó mediante la adición de tres muestras de aceite de semillas de Persea americana Miller variedad Lorena, con un patrón de 100 ppm de estigmasterol, realizado por triplicado, luego se comparó con un triplicado sin adición.

\section{Parámetros instrumentales}

El equipo utilizado para la estandarización fue un Cromatógrafo de Gases con detector de ionización de llama marca Shimadzu GC-2014, equipado con autoinyector AOC$20 \mathrm{i}+\mathrm{s}$, automuestrador $\mathrm{AOC}-2 \mathrm{Oi}+\mathrm{s}$, inyección Split y columna MXT-5 serie 951048 (30 m de longitud $\times 0.5 \mathrm{~mm} \mathrm{ID,} 0.5 \mu \mathrm{m}$ de espesor de película).

Las condiciones que se establecieron para los análisis cromatográficos fueron: gas de arrastre Helio, flujo total 45 $\mathrm{mL} / \mathrm{min}$, flujo en la columna $4 \mathrm{~mL} / \mathrm{min}$, velocidad lineal 74 
$\mathrm{cm} / \mathrm{s}$, temperatura del inyector $300^{\circ} \mathrm{C}$, volumen de inyección $1 \mu \mathrm{L}$, modo de inyección plit, radio plit 10, temperatura de la columna $250^{\circ} \mathrm{C}$ durante 2 minutos, después se llevó hasta $280^{\circ} \mathrm{C}$ con razón $40^{\circ} \mathrm{C} / \mathrm{min}$, se mantuvo así durante 10 minutos, después se llevó hasta $300^{\circ} \mathrm{C}$ con razón de $10^{\circ} \mathrm{C} / \mathrm{min}$ y se mantuvo por 5 minutos, como detector se empleó un detector de ionización de llama (DIL) a una temperatura de $360^{\circ} \mathrm{C}$ (Arias y Gil, 2012).

La confirmación se realizó usando un Cromatógrafo de Gases (Agilent Technologies modelo 7890 ) acoplado a espectrometría de masas (Agilent Technologies modelo 5975C) con una columna (modelo DB-5ms) de $60 \mathrm{~m}$ de longitud $\times 320 \mu \mathrm{m}$ ID, $0.25 \mu \mathrm{m}$ de espesor de película. La fase móvil fue gas helio con un flujo constante de $65 \mathrm{~mL} / \mathrm{min}$ y 12.561 de presión, la temperatura del inyector fue de $250^{\circ} \mathrm{C}$, volumen de inyección $5 \mu \mathrm{L}$, modo de inyección splitless, la temperatura inicial de la columna fue de $50^{\circ} \mathrm{C}$ que se mantuvo durante 1 minuto, después se llevó hasta $125^{\circ} \mathrm{C}$ a razón de $25^{\circ} \mathrm{C} / \mathrm{min}$, finalmente se llevó a $300^{\circ} \mathrm{C}$ a razón de $10^{\circ} \mathrm{C} /$ min y se mantuvo así durante 7 minutos.

Para la cualificación de ergosterol se utilizó un Cromatógrafo Líquido de Alta Eficiencia (CLAE) HITACHI LaChrom; bomba L-2130; horno L-2300; detector UV-Vis L2420, con una columna Ultra AQ C18 ( $3 \mu \mathrm{m} \times 100 \mathrm{~mm} \times 3.2$ $\mathrm{mm}$ ) marca Restek, a temperatura ambiente, como fase móvil se utilizó una mezcla metanol/agua (80:20), flujo de 0.3 $\mathrm{mL} / \mathrm{min}$, volumen de inyección de $20 \mu \mathrm{L}$ y longitud de onda de $280 \mathrm{~nm}$ (Yuan et al., 2007).

\section{Concentración de fitoesteroles}

Con la mezcla de los estándares de fitoesteroles, se hicieron varias diluciones y se establecieron seis diluciones (tabla 2).

Tabla 2. Diluciones de los estándares de fitoesteroles.

\begin{tabular}{lcccccc} 
& \multicolumn{7}{c}{ diluciones } \\
\cline { 2 - 7 } Estándar & $\mathbf{1}$ & $\mathbf{2}$ & $\mathbf{3}$ & $\mathbf{4}$ & $\mathbf{5}$ & 6 \\
Estigmasterol & 5 & 15 & 30 & 75 & 150 & 300 \\
5 a-Colestano & 5 & 15 & 30 & 75 & 150 & 300 \\
\hline
\end{tabular}

Con las anteriores diluciones, se construyeron las respectivas curvas de calibración para cada estándar y se calcularon los parámetros estadísticos: precisión, límite de detección y cuantificación, sensibilidad y linealidad.

\section{RESULTADOS Y DISCUSIÓN |}

\section{Extracción de aceite}

El aceite obtenido de las semillas de Persea americana Miller variedad Lorena presentó una coloración café, con una consistencia sólida (Fig. 4) y un porcentaje de rendimiento de extracción de $8.47 \%$.

\section{Extracción de la materia insaponificable}

La materia insaponificable obtenida del aceite de semillas de Persea americana Miller variedad Lorena presentó una coloración café y un aspecto sólido (Fig. 5) con un porcentaje de rendimiento de $76.9 \%$.

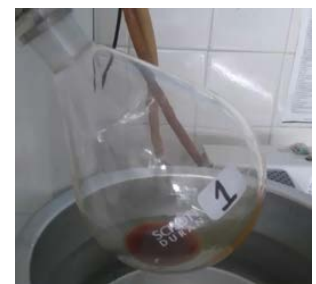

Figura 4. Aceite de las semillas de Persea americana Miller variedad Lorena.

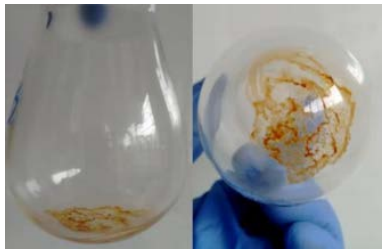

Figura 5. Materia insaponificable del aceite de semillas de Persea americana Miller variedad Lorena.

\section{Porcentaje de recuperación}

En la tabla 3 se presentan los resultados de los porcentajes de recuperación obtenidos para el estigmasterol en las muestras de aceite de semillas de Persea americana Miller var. Lorena.

Tabla 3. Datos de porcentaje de recuperación para el estándar de estigmasterol

\begin{tabular}{|c|c|c|}
\hline $\begin{array}{c}\text { Muestra de aceite } \\
(n=3)\end{array}$ & $\begin{array}{c}\text { Concentración del estándar } \\
(\mathrm{ppm})\end{array}$ & $\begin{array}{l}\text { Porcentaje de } \\
\text { recuperación }\end{array}$ \\
\hline 1 & 100 & 86.45 \\
\hline 2 & 100 & 88.24 \\
\hline 3 & 100 & 83.96 \\
\hline \multirow{2}{*}{\multicolumn{2}{|c|}{$\begin{array}{c}\text { Media } \\
\text { Desviación estándar }\end{array}$}} & 86.22 \\
\hline & & 1.755 \\
\hline \multicolumn{2}{|r|}{$\% \mathrm{CV}$} & 2.036 \\
\hline
\end{tabular}

De acuerdo con los resultados (tabla 3), la saponificación asistida por microondas tuvo un buen rendimiento para la obtención de materia insaponificable y los sistemas de elución para la extracción en fase sólida (SPE) obtuvo buenos porcentajes de recuperación para el estándar de estigmasterol, con un promedio de $86.22 \%$. Al comparar con la literatura científica, se encuentra que los porcentajes de recuperación de algunos fitoesteroles varían entre 80$100 \%$, un ejemplo de esto es el aceite de oliva, en el cual se han reportado porcentajes de recuperación entre 70-110\% para el estigmasterol y $\beta$-sitoesterol respectivamente (Bernal y Díaz, 2008; Polania, 2014). Por lo anterior se adaptó dicho método de recuperación que se acercan mucho a los encontrados en estudios previos.

\section{Análisis cualitativo}

Para la identificación de ergosterol se utilizó el método implementado por Cromatografía Líquida de Alta Eficiencia ( $C L A E)$, con base en las condiciones descritas anteriormente, en la figura 6 se puede observar el cromatograma del estándar de ergosterol (300 ppm) y el de la fracción de fitoesteroles, los cuales eluyen en un tiempo de retención 
similar (3.953 min para el estándar con un área de 8916309 y 4.217 min para la muestra con un área de 609051243), lo que permite establecer la presencia de este fitoesterol en semillas de Persea americana Miller variedad Lorena, además la concentración del ergosterol es superior a la concentración del estándar, por lo tanto es uno de los fitoesteroles más abundantes en la semilla de Persea americana Miller variedad Lorena.
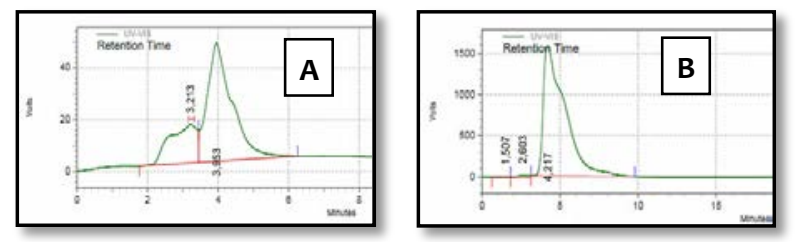

Figura 6. Cromatogramas obtenidos por CLAE. A). Estándar (300 ppm) y B). Fracción de fitoesteroles.

Para la identificación de otros fitoesteroles se inyectó al cromatógrafo de gases con detector de ionización de llama (GC-FID) una mezcla de tres estándares de fitoesteroles (Fig. 7) para comparar los tiempos de retención con la fracción de fitoesteroles (Fig. 8) obtenida del aceite de las semillas de Persea americana Miller variedad Lorena, los resultados obtenidos se observan en la tabla 4:

Tabla 4. Tiempo de retención de los compuestos de referencia evaluados y los compuestos presentes en la fracción de fitoesteroles

\begin{tabular}{ccc}
\hline Compuesto & \multicolumn{2}{c}{ Tiempo de retención $(\min )$} \\
& Estándar & Fracción de fitoesteroles \\
\hline 5-a-colestano & 6.429 & 6.314 \\
colestanol & 9.826 & 9.619 \\
estigmasterol & 12.184 & 12.168 \\
\hline
\end{tabular}

De acuerdo con lo anterior se identificaron los tres fitoesteroles en la muestra y se realizaron las curvas de calibración para los estándares de 5-a-colestano y estigmasterol para la posterior cuantificación en la muestra.

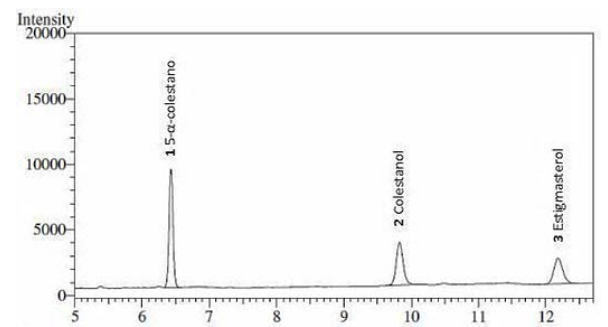

Figura 7. Cromatograma de la mezcla de estándares de fitoesteroles en GC-FID.

Para la confirmación y análisis de los fitoesteroles presentes se realizó un ensayo por medio de cromatografía de gases acoplado a espectrometría de masas (CG-EM), cuyos resultados se analizaron por comparación de sus espectros de masas con los reportados por la biblioteca NIST. En la tabla 5, se observan los compuestos identificados.

De acuerdo a la información (tabla 5) se confirmó la presencia de fitoesteroles en el aceite de la semilla de Persea americana Miller variedad Lorena, porque la fracción de fitoesteroles contiene derivados de 5 - $\alpha$-colestano.

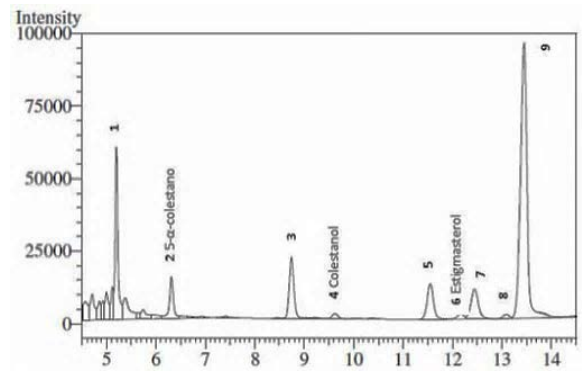

Figura 8. Cromatograma de la fracción de fitoesteroles en GC-FID

Tabla 5. Análisis composicional por cromatografía de gases acoplado a espectrometría de masas de la fracción de fitoesteroles.

\begin{tabular}{cc}
\hline Nombre & Fórmula \\
\hline $5 \alpha$-acetiloxi-3 $\beta$-hidroxicolestano & $\mathrm{C}_{29} \mathrm{H}_{50} \mathrm{O}_{3}$ \\
$5,6 \alpha$-epoxicolestano & $\mathrm{C}_{27} \mathrm{H}_{46} \mathrm{O}$ \\
\hline
\end{tabular}

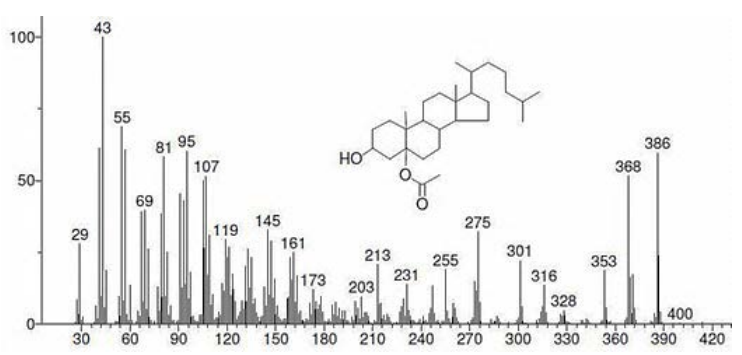

Figura 9. Espectro de masas del compuesto $5 \alpha$-acetiloxi-3 $\beta$ hidroxicolestano obtenido de la fracción de fitoesteroles.
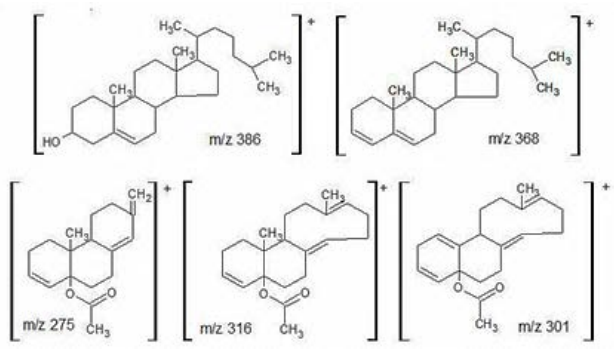

Figura 10. Posibles fragmentos del compuesto $5 \alpha$-acetiloxi-3 $\beta$ hidroxicolestano

En la figura 9, se presenta el espectro de masas del compuesto $5 \alpha$-acetiloxi-3 $\beta$-hidroxicolestano, fórmula molecular $\mathrm{C}_{29} \mathrm{H}_{50} \mathrm{O}_{3}$ con una probabilidad de $9.89 \%$, y un tiempo de retención de $27.235 \mathrm{~min}$, un ion molecular de $\mathrm{m} / \mathrm{z}$ 446 y un pico base $\mathrm{m} / \mathrm{z}$ 43. Según el espectro de masas del compuesto (Fig. 9) y sus posibles fragmentos (Fig. 10) presenta iones importantes con valores m/z: 386 y 368 que indican que este compuesto puede ser un esterol. Los fragmentos con valores de m/z: 386 (perdida del éster), $\mathrm{m} / \mathrm{z}$ 368 (perdida del éster y agua), m/z 334 (perdida de la cadena lateral), m/z 316 (perdida de la cadena lateral y agua), m/z 301 (perdida de la cadena lateral, agua y un grupo metil), m/z 293 
(fisión del anillo D) y m/z 275 (fisión del anillo D - agua) confirman la presencia de esteroles en la fracción de fitoesteroles de la materia insaponificable del aceite.

\section{Análisis cuantitativo}

Se determinó el contenido de fitoesteroles en la fracción de fitoesteteroles de la materia insaponificable del aceite de las semillas de Persea americana Miller variedad Lorena, con base en el método anteriormente planteado para Cromatografía de Gases, posteriormente se inyectaron los patrones de menor a mayor concentración y luego las muestras. En la tabla 6 se muestran las mediciones de los patrones para el estándar de estigmasterol.

Tabla 6. Datos cromatográficos para diferentes concentraciones (ppm) para el estigmasterol.

\begin{tabular}{ccccccc}
\hline Concentración (ppm) & $\mathbf{5}$ & $\mathbf{1 5}$ & $\mathbf{3 0}$ & $\mathbf{7 5}$ & $\mathbf{1 5 0}$ & $\mathbf{3 0 0}$ \\
\hline Área & 4691 & 15909 & 31877 & 80619 & 173636 & 367269 \\
$\mathbf{n}=\mathbf{3}$ & 4358 & 15994 & 32779 & 80758 & 180439 & 387898 \\
$\% \mathrm{CV}$ & 4567 & 16047 & 32493 & 80406 & 173153 & 366980 \\
\hline
\end{tabular}

Según la tabla 6, los valores de coeficiente de variación estándar (CV) son inferiores al $4 \%$ en los tres niveles de concentración estudiados, lo que indica una buena reproducibilidad del método.

\section{Estandarización de la técnica cromatográfica}

Se elaboró la curva de calibración para los estándares de estigmasterol y 5-a-colestano, en la figura $\mathbf{1 1}$ se puede observar la curva de calibración para el estigmasterol.

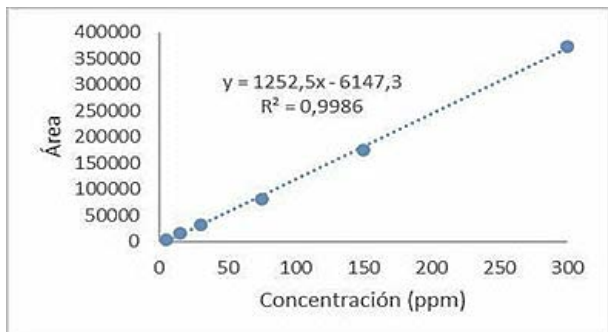

Figura 11. Curva de calibración del estigmasterol.

Los datos estadísticos analizados fueron coeficiente de correlación $\left(R^{2}\right)$, Coeficiente de variación estándar (CV), Límite de detección (LD), Límite de cuantificación (LC) y Sensibilidad (S), los cuales se pueden observar en la tabla 6.

Tabla 7. Resultados estadísticos para las curvas de calibración.

\begin{tabular}{cccccc}
\hline Estándar & $\mathbf{R}^{2}$ & $\mathbf{C V}$ & $\begin{array}{c}\text { LD } \\
(\mathbf{p p m})\end{array}$ & $\begin{array}{c}\text { LC } \\
(\mathrm{ppm})\end{array}$ & $\begin{array}{c}\mathbf{S} \\
(\mathrm{ppm} / \text { área })\end{array}$ \\
\hline Estigmasterol & 0.9986 & 2.009 & 0.4556 & 1.5186 & 834.6 \\
5-a-colestano & 0.9996 & 1.992 & 0.8331 & 2.7769 & 743.8 \\
\hline
\end{tabular}

Como se observa en la tabla 7, los coeficientes de correlación $\left(R^{2}\right)$ fueron en promedio de 0.999; indicando una buena linealidad y una correlación adecuada entre las variables (Piironen et al., 2000), la precisión del método evaluada con el coeficiente de variación (CV) se encuentra dentro de los valores aceptados (menores al 5\%) indicando una buena precisión, reproducibilidad y repetibilidad; los límites detección (LD) obtenidos se encuentran en un rango de 0.4556 y 0.8331 ppm y los límites de cuantificación (LC) entre 1.5186 y 2.7769 ppm, estos valores son adecuados, debido a que los esteroles vegetales constituyen la mayor porción de la materia insaponificable de los aceites vegetales (Du y Ahn, 2002), por lo tanto, el método tiene la capacidad de detectar y cuantificar fitoesteroles a muy bajas concentraciones en matrices oleosas. El valor de sensibilidad está asociado al valor de la pendiente de las curvas de calibración (Skoog et al., 2008), como se aprecia en la tabla 7 , la sensibilidad de cada una de las curvas de calibración fue buena debido a pendientes considerables, lo que indica que el método detecta los mínimos cambios de concentración del analito en las muestras.

En la tabla 8 se encuentran todos los resultados obtenidos desde el tratamiento de la muestra hasta la cuantificación de los fitoesteroles (5-a-colestano y estigmasterol), con valores estadísticos como la media, desviación estándar y coeficiente de variación (CV).

Según los resultados obtenidos en la tabla 8 , se pudo establecer que la saponificación asistida por ultrasonido es un método adecuado para la obtención de materia insaponificable (MI), debido a que se obtuvo un alto porcentaje de rendimiento (76.9\%), además, la cantidad de muestra a utilizar puede ser entre 10 y $100 \mathrm{mg}$ y el tiempo de saponificación no tarda más de 15 minutos comparado con otros métodos como el de saponificación por reflujo, que puede demorar entre 30 min y 3 horas.

Tabla 8. Resultados obtenidos de porcentaje de recuperación y cuantificación de fitoesteroles.

\begin{tabular}{ccccc}
\hline Réplicas & $\begin{array}{c}\text { Rendimiento } \\
\text { de aceite }(\%)\end{array}$ & $\begin{array}{c}\text { Rendimiento } \\
\text { de } \mathbf{M I}(\%)\end{array}$ & $\begin{array}{c}\text { Concentración } \\
\text { de 5a-colestano } \\
\text { (ppm) }\end{array}$ & $\begin{array}{c}\text { Concentración de } \\
\text { estigmasterol } \\
\text { (ppm) }\end{array}$ \\
\hline $\mathbf{1}$ & 7.52 & 75.70 & 49.77 & 17.74 \\
$\mathbf{2}$ & 9.23 & 76.42 & 50.70 & 19.92 \\
$\mathbf{3}$ & 8.67 & 78.57 & 48.83 & 19.84 \\
$\begin{array}{c}\text { Media } \\
\text { Desviación } \\
\text { estándar }\end{array}$ & 8.47 & 76.90 & 49.77 & 19.17 \\
CV & 0.712 & 1.219 & 0.763 & 1.009 \\
\hline
\end{tabular}

\section{CONCLUSIONES}

El aceite de semillas de Persea americana Miller variedad Lorena tiene porcentaje de rendimiento de $8.47 \%$, un porcentaje de materia insaponificable de $76.9 \%$ y la recuperación de esteroles a partir de este aceite por la técnica de extracción en fase sólida (SPE), arrojó un porcentaje de recuperación de 86.22 \% indicando una adecuada recuperación de los analitos.

Se estandarizó la técnica cromatografía de gases con detector de ionización de llama para el análisis de fitoesteroles, mostrando valores estadísticos adecuados para la cuantificación de estos; con coeficientes de correlación $\left(\mathrm{R}^{2}\right)$ cercanos a la unidad, los límites de detección y cuantificación por debajo de 3 ppm, valores de sensibilidad altos y una precisión con valores de coeficiente de variación inferiores al $5 \%$ indicando la precisión, reproducibilidad y 
repetibilidad de método. Demostrando así la validez de la técnica para el análisis de fitoesteroles.

Las semillas de Persea americana Miller variedad Lorena contiene como fitoesterol mayoritario el ergosterol el cual se determinó de forma cualitativa por CLAE, mientras que por CG-FID se cuantificó el 5a-colestano con una concentración de $49.77 \mathrm{mg}$ por $\mathrm{Kg}$ de aceite, seguido de estigmasterol con una concentración de 19.17 mg de estigmasterol por Kg de aceite..

\section{AGRADECIMIENTOS}

Al Grupo de Investigación de Oleoquímica de la Universidad Tecnológica de Pereira por la financiación del trabajo de investigación.

\section{REFERENCIAS}

- Abidi S.L. 2001. Chromatographic analysis of plant sterols in foods and vegetable oils. Journal of Chromatography, 935: 173-201.

- Arias A y Gil D. 2012. Estandarización de la técnica cromatografía de gases capilar para la identificación y cuantificación de fitoesteroles en semillas de luffa cylindrica. Trabajo de grado, Programa de Química Industrial, Facultad de Tecnologías, Escuela de Química, Tecnológica de Pereira, Pereira, $80 \mathrm{p}$.

- Awad A.B. y Fink C.S. 2000. Phytosterols as anticancer dietary components: evidence and mechanism of action. Journal of Nutrition, 130(9): 2127-2130.

- Azadmard S., Savage G y Dutta P. 2005. Sterol fractions in hazelnut and virgin olive oils and 4, 40 -dimethylsterols as possible markers for detection of adulteration of virgin olive oil. Journal of the American Oil Chemists' Society, 82: 717-725.

- Bernal J. y Díaz, C. 2008. Tecnología para el cultivo del Aguacate: Generalidades del cultivo. Manual técnico 5. Corporación Colombiana de Investigación Agropecuaria, CORPOICA. Centro de Investigación La Selva, Rio Negro, Antioquía. 241 p.

- Brufau G, Canela MA y Rafecas M. 2008. Phytosterols: physiologic and metabolic aspects related to cholesterollowering properties. Nutrition Research, 28(4): 217-225.

- Clement LM, Hansen SL, Costin, CD y Perri, GL. 2010. Quantitation of sterols and steryl esters in fortified foods and beverages by GC/FID. Journal of the American Oil Chemists' Society, 87: 973-980.

- Du M y Ahn, DU. 2002. Simultaneous analysis of tocopherols, cholesterol, and phytosterols using. Journal of Food Chemistry and Toxicology Simultaneous, 67(5):1696-1700.

- Gaona G. 2011. Estudio del efecto en el perfil lipídico y adipocitocinas, tras el consumo sostenido de aceite de aguacate, de semilla de uva y de soya, en ratas. Trabajo de grado, Facultad de Ciencias Naturales, Universidad Autónoma de Querétaro, México, 74 p.

- Harrabi S, St-Amand A, Sakouhi F, Sebei K, Kallel H, Mayer PM y Boukhchina S. 2008. Phytostanols and phytosterols distributions in corn kernel. Food Chemistry, 111: 115-120.

- Howard LR, Clark JR y Brownmiller C. 2003. Antioxidant capacity and phenolic content in blueberries as affected by genotype and growing season. Journal of the Science of Food and Agriculture, 83: 1238-1247.

- Lagarda MJ, García-Llatas G y Farré R. 2006. Analysis of phytosterols in foods. Journal of Pharmaceutical and Biomedical Analysis, 41(5): 1486- 1496.

- Michelle AM y Manohar LG. 2009. Anti-inflammatory and cardioprotective effects of $n-3$ polyunsaturated fatty acids and plant sterols in hyperlipidemic individuals. Atherosclerosis, 204(2):476-482.
- Moghadasian MH y Frohlich JJ. 1999. Effects of dietary phytosterols on cholesterol metabolism and atherosclerosis: clinical and experimental evidence. The American Journal of Medicine, 107(6): 588-594.

- Moreau RA y Hicks KB. 2005. The composition of corn oil obtained by the alcohol extraction of ground corn. Journal of the American Oil Chemists' Society, 82: 809-815.

- Moreau RA, Whitaker BD y Hicks KB. 2002. Phytosterols, phytostanols, and their conjugates in foods: structural diversity, quantitative analysis, and health-promoting uses. Progress in Lipid Research, 41(6): 457- 500.

- Nyström L, Mäkinen M, Lampi AM y Piironen V. 2005. Antioxidant Activity of Steryl Ferulate Extracts from Rye and Wheat Bran. Journal of Agricultural and Food Chemistry, 53(7): 2503-2510.

- Ozdemir F y Topuz, A. 2004. Changes in dry matter, oil content and fatty acids composition of avocado during harvesting time and post-harvesting ripening period. Food Chemistry, 86: 79-83.

- Piironen V, Lindsay DG, Miettinen TA, Toivo J y Lampi AM. 2000. Plant sterols: biosynthesis, biological function and their importance to human nutrition. Journal of the Science of Food and Agriculture, 80(7): 939-966.

- Polania W. 2014. Actividad antioxidante de los residuos del aguacate Hass (Persea americana Mill. var Hass) sometidos a extracciones clásicas y a fluidos presurizados. Trabajo de grado, Facultad de Ciencias, Departamento de Química, Universidad Nacional de Colombia, Bogotá, D.C., 103 P

- Rodríguez J, Amaya C, Caballero P, Alanís G, Aguilera C, Báez J, Moreno S y Núñez A. 2015. Factors affecting the content of squalene, total and esterified phytoesterols in soybean distillated fatty acids by-product for its potential use. Nova Scientia, 7(2):268-285.

- Santos R, Limas E, Sousa M, Castilho M.d.C, Ramos F y Noronha, d.S.M.I. 2007. Optimization of analytical procedures for GC-MS determination of phytosterols and phytostanols in enriched milk and yoghurt. Food Chemistry, 102: 113-117.

- Skoog DA, Holler FJ y Crouch SR. 2008. Principios de análisis instrumental, 6th ed., Cengage Learning Editores, México, $1064 \mathrm{p}$.

- Slavin M y Yu LL. 2012. A single extraction and HPLC procedure for simultaneous analysis of phytosterols, tocopherols and lutein in soybeans. Food Chemistry, 135 (4): 2789-2795.

- Smania EF, Delle Monache F, Smania A, Yunes RA y Cuneo RS. 2003. Antifungal activity of sterols and triterpenes isolated from Ganoderma annulare. Fitoterapia, 74(4): 375-377.

- Thomas RH, Woods FM, Dozier WA, Ebel, RC, Nesbitt M, Wilkins B y Himelrick DG. 2005. Cultivar variation in physicochemical and antioxidant activity of Alabama-grown blackberries. Small Fruits, 4: 57-71.

- Toivo J, Phillips K, Lampi AM y Piironen, V. 2001. Determination of sterols in foods: recovery of free, esterified, and glycosidic sterols. Journal of Food Composition and Analysis, 14: 631-643.

- Valenzuela B. y Ronco AM. 2004. Fitoesteroles y Fitoestanoles: Aliados naturales para la protección de la salud cardiovascular. Revista Chilena de Nutrición, 21: 161-169.

- Verleyen T, Forcades M, Verhe R, Dewettinck K, Huyghebaert A y De G.W. 2002. Analysis of free and esterified sterols in vegetable oils. Journal of the American Oil Chemists' Society, 79: 117-122.

- Wanga T, Hicks KB y Moreau R. 2002. Antioxidant activity of phytosterols, oryzanol, and other phytosterol conjugates. Journal of the American Oi Chemists' Society, 79(12): 1201-1206.

- Young C. 1995. Microwave-Assisted Extraction of the Fungal Metabolite Ergosterol and Fatty Acids. Journal of Agricultural and Food Chemistry, 43: 2904-2910.

- Yuan J, Wang J, Liu X, Kuang H y Zhao S. 2007. Simultaneous determination of free ergosterol and ergosteryl esters in Cordyceps sinensis by HPLC. Food Chemistry, 105: 1755-1759. 